Check for updates

Cite this: RSC Adv., 2019, 9, 20375

\title{
Fabrication of plasmonic dye-sensitized solar cells using ion-implanted photoanodes $\uparrow$
}

\author{
Navdeep Kaur, ${ }^{a}$ Aman Mahajan, (D) *a Viplove Bhullar, ${ }^{\text {a }}$ Davinder Paul Singh, ${ }^{a}$ \\ Vibha Saxena, ${ }^{b}$ A. K. Debnath, ${ }^{\text {b }}$ D. K. Aswal, ${ }^{c}$ Devarani Devi, ${ }^{d}$ Fouran Singh ${ }^{d}$ \\ and Sundeep Chopra ${ }^{d}$
}

Plasmonic dye-sensitized solar cells containing metal nanoparticles suffer from stability issues due to their miscibility with liquid iodine-based electrolytes. To resolve the stability issue, herein, an ion implantation technique was explored to implant metal nanoparticles inside $\mathrm{TiO}_{2}$, which protected these nanoparticles with a thin coverage of $\mathrm{TiO}_{2}$ melt and maintained the localized surface plasmon resonance oscillations of the metal nanoparticles to efficiently enhance their light absorption and make them corrosion resistant. Herein, Au nanoparticles were implanted into the $\mathrm{TiO}_{2}$ matrix up to the penetration depth of $22 \mathrm{~nm}$, and their influence on the structural and optical properties of $\mathrm{TiO}_{2}$ was studied. Moreover, plasmonic dye-sensitized solar cells were fabricated using N719 dye-loaded Au-implanted $\mathrm{TiO}_{2}$ photoanodes, and their power conversion efficiency was found to be $44.7 \%$ higher than that of the unimplanted $\mathrm{TiO}_{2}$-based dye-sensitized solar cells due to the enhanced light absorption of the dye molecules in the vicinity of the localized surface plasmon resonance of $\mathrm{Au}$ as well as the efficient electron charge transport at the $\mathrm{TiO}_{2}$ (aAuaN719/electrolyte interface.

Received 9th April 2019

Accepted 14th June 2019

DOI: $10.1039 / c 9 r a 02657 f$

rsc.li/rsc-advances enhance their PCE by improving light absorption along with fast electron charge transfer from the dye molecules to $\mathrm{TiO}_{2}{ }^{18-20}$ and inhibiting the back recombination of electrons from either the dye molecules or the conduction band (C.B.) of $\mathrm{TiO}_{2}$ to the electrolyte. ${ }^{21,22}$ Chen et al. $^{23}$ optimized $\mathrm{TiO}_{2}$-based DSSCs incorporated with $0.8 \mathrm{wt} \%$ of $\mathrm{Au} \mathrm{nps}$ (200-300 nm size) and showed a $12.2 \%$ increase in the PCE as compared to the case of $\mathrm{TiO}_{2}$-based DSSCs due to enhanced light absorption induced by the Au nps. Muduli et al. ${ }^{24}$ reported a 16\% increase in the PCE of $\mathrm{TiO}_{2}-\mathrm{Au}$-based DSSCs owing to fast electron transport and reduced recombination of electrons and holes at the $\mathrm{TiO}_{2} @ \mathrm{Au}$ / dye/electrolyte interface. Chander et $a .^{25}$ showed an increment of $19.12 \%$ in the PCE of $\mathrm{TiO}_{2}$-based DSSCs loaded with $0.24 \mathrm{wt} \%$ Au nps (36 nm size). Zhao et al. ${ }^{26}$ demonstrated that the incorporation of $0.8 \mathrm{wt} \% \mathrm{Ag}$ into the $3 \mathrm{D}$ hierarchical microstructure of $\mathrm{TiO}_{2}$ led to a $30.7 \%$ enhancement in the PCE of plasmonic DSSCs due to their increased light scattering ability. The incorporation of $\mathrm{Ag}$ nanowires into $\mathrm{TiO}_{2}$ has been found to enhance the light absorption and electron charge transport of DSSCs, resulting in a $25.3 \%$ enhancement of their $\mathrm{PCE}^{27} \mathrm{Li}$ et al. reported the incorporation of $0.168 \mathrm{wt} \%$ of $\mathrm{Au}$ into $\mathrm{TiO}_{2}$ based DSSCs, which exhibited a maximum $45 \%$ increment in the PCE. ${ }^{28}$ Thus, the variation in the amount of Mnps incorporated into $\mathrm{TiO}_{2}$ has been found to significantly affect the performance of plasmonic DSSCs.

Although these research findings show an improvement in PCE, the stability of the bare Mnp-incorporated DSSCs has not been discussed to date. Moreover, the bare $\mathrm{Mnps}$ in $\mathrm{TiO}_{2}$ are in

\footnotetext{
a Department of Physics, Guru Nanak Dev University, Amritsar-143 005, India. E-mail: aman.phy@gndu.ac.in

${ }^{b}$ Thin Film Devices Section, Technical Physics Division, Bhabha Atomic Research Centre, Mumbai - 400 085, India

${ }^{c}$ National Physical Laboratory, New Delhi - 110012, India

${ }^{d}$ Inter University Accelerator Centre, New Delhi - 110 0067, India

$\dagger$ Electronic supplementary information (ESI) available. See DOI: 10.1039/c9ra02657f
} 
direct contact with the dye molecules and electrolyte; this results in the recombination of photo-generated carriers and the corrosion of the Mnps, respectively. This further reduces the PCE as well as the stability of the plasmonic DSSCs. ${ }^{29,30}$ Standridge et $a l .{ }^{31}$ reported the corrosion of $\mathrm{Ag}$ nps in the presence of a redox electrolyte in plasmonic DSSCs; this reduced the photocurrent and hence the PCE. Similar observations have been made in our laboratory where higher $\mathrm{PCE}$ of the $\mathrm{TiO}_{2} @ \mathrm{Ag}$ based DSSC was obtained initially, followed by a reduction in the PCE after some time due to the corrosion of the Mnps with the redox $\mathrm{I}^{-} / \mathrm{I}_{3}{ }^{-}$electrolyte. Thus, a spacer layer needs to be inserted in between the Mnps and the electrolyte while fabricating highly stable DSSCs to prevent the corrosion of the Mnps; in this direction, a shell of silica $\left(\mathrm{SiO}_{2}\right)$ and $\mathrm{TiO}_{2}$ coated on the Mnp core has been investigated; moreover, Choi et al. ${ }^{32}$ have observed that the DSSCs incorporated with Au-capped $\mathrm{TiO}_{2}$ and $\mathrm{SiO}_{2}$ exhibit a $9.0 \%$ and $5.0 \%$ increment in the PCE, respectively. The PCE increment of $16 \%$ has been observed in a AuNR@SiO ${ }_{2}$-incorporated plasmonic tandem DSSC due to its increased light-harvesting ability from the visible to the nearinfrared region. ${ }^{33}$ Bai et al. ${ }^{34}$ have reported $\mathrm{SiO}_{2}$-capped $\mathrm{Au}$ nanorod-based plasmonic DSSCs that have exhibited a $23 \%$ enhancement in the PCE. However, the insulating nature of $\mathrm{SiO}_{2}$ opposes the injection of photo-generated carriers into $\mathrm{TiO}_{2}$ and results in the poor stability of plasmonic DSSCs. Moreover, the separation between the dye molecules and Mnps adversely affects the strength of the electromagnetic field. The increased separation has led to a decrease in the plasmon-enhanced photocurrent in DSSCs. Standridge et al. ${ }^{35}$ have reported that the threshold thickness of a few nm of the $\mathrm{TiO}_{2}$ shell around the $\mathrm{Ag}$ nps is required to effectively protect these nps from the longtime exposure to the aggressive $\mathrm{I}^{-} / \mathrm{I}_{3}{ }^{-}$environment; the $\mathrm{Au} @ \mathrm{TiO}_{2}$ hollow submicrosphere (with controllable sizes and shell thicknesses)-based plasmonic DSSCs have revealed a 30\% increment in the PCE due to their increased light absorption. ${ }^{36}$ $\mathrm{Xu}$ et $a l .{ }^{37}$ have reported a $16 \%$ increase in the PCE of DSSCs containing core-shell popcorn-shaped Au-Ag alloy nps in $\mathrm{TiO}_{2}$ photoanodes. Although the stability of the plasmonic DSSC has been found to improve using core-shell Mnps, the PCE has not been significantly enhanced.

Thus, a novel technique of ion implantation has been explored to make the utmost use of the LSPR property of Mnps by preventing them from corrosion as well as coming in direct contact with the dye molecules, which not only provides stability to the DSSCs but also makes them more efficient. Ion implantation modifies the properties of $\mathrm{TiO}_{2}$ and provides a controllable growth of the Mnps at different depths inside the $\mathrm{TiO}_{2}$ protected with a thin layer of $\mathrm{TiO}_{2}$ melt. ${ }^{38-40}$ However, only a few studies have been reported on the ion implantation of Mnp-based plasmonic DSSCs. Luo et al. have reported the effect of $\mathrm{Ag}$ implantation on the performance of DSSC and found a nearly $25 \%$ enhancement in the PCE. ${ }^{41}$

In this study, the $\mathrm{Au} \mathrm{Mnps}$ were implanted inside a $\mathrm{TiO}_{2}$ matrix for the first time for the fabrication of stable and efficient plasmonic DSSCs; the fluence of the ion beam was optimized for balancing the LSPR of the Au nps and porosity of $\mathrm{TiO}_{2}$ to fabricate improved plasmonic DSSCs; moreover, it was found that the Au-implanted plasmonic DSSCs exhibited the maximum PCE enhancement of $44.7 \%$. A plausible working mechanism of the stable and efficient plasmonic DSSCs was explained on the basis of the thermal spike model and spectroscopic and electrochemical investigations.

\section{Experimental}

Fluorine-doped tin oxide (FTO) glass substrate (sheet resistance of $\sim 7 \Omega \mathrm{sq}^{-1}$ ), ethanol, soap solution, isopropanol, zinc powder, hydrochloric acid, titanium(Iv) isopropoxide (TTIP), platinum (Pt) paste and the di-tetrabutylammonium cis-bis(isothiocyanato)bis(2,2'-bipyridyl-4,4'-dicarboxylato)ruthenium(II) (N719) dye of analytical grade were procured from Sigma Aldrich. Titanium dioxide $\left(\mathrm{TiO}_{2}\right)$ paste and iodide-tri iodide $\mathrm{I}^{-} / \mathrm{I}_{3}{ }^{-}$redox electrolyte in 3-methoxypropionitrile (EL-HSE) were procured from Dyesol, Australia. The photoanodes were prepared by spincoating a compact layer of the TTIP solution onto the patterned FTO substrates followed by annealing at $450{ }^{\circ} \mathrm{C}$ for $30 \mathrm{~min}$. The $\mathrm{TiO}_{2}$ paste was deposited onto the annealed films using a doctor blade technique with the thickness of $3 \mu \mathrm{m}$ and then subjected to thermal annealing at $450{ }^{\circ} \mathrm{C}$ for 30 min under air atmosphere. The $\mathrm{Au}$ ion beam was implanted onto the $\mathrm{TiO}_{2}$ matrix with the energy of $80 \mathrm{keV}$ under high vacuum conditions at room temperature using a low-energy negative-ion implanter (Inter University Accelerator Centre (IUAC), New Delhi, India) at different fluence values of $1 \times 10^{13}, 1 \times 10^{14}, 1 \times 10^{15}$ and $1 \times$ $10^{16}$ ions $\mathrm{cm}^{-2}$, and the obtained samples were named F1, F2, F3 and F4, respectively. The penetration depth of the Au nps in $\mathrm{TiO}_{2}$ was found to be $22 \mathrm{~nm}$ using the Stopping and Range of Ion in Matter (SRIM) software.

Prior to the fabrication of a cell, the unimplanted and implanted $\mathrm{TiO}_{2}$ were soaked in a $0.3 \mathrm{mM}$ solution of N719 dye for $24 \mathrm{~h}$ under dark conditions. The counter electrode (CE) was prepared by doctor blading Pt onto the pre-cleaned FTO substrates followed by annealing for $30 \mathrm{~min}$ at $450{ }^{\circ} \mathrm{C}$ in air. The DSSCs were assembled using prepared photoanodes and a Pt $\mathrm{CE}$ in a sandwich-type geometry along with the insertion of an electrolyte in them.

The surface morphology of the samples was studied via a field emission scanning electron microscope (FESEM-Carl Zeiss, Supra 55). The X-ray photoelectron spectroscopy (XPS) measurements of the samples were conducted using the MAC2 electron analyzer system connected to a MBE machine (EVA-32 Riber, France) with the excitation source of $\mathrm{Mg} \mathrm{K}_{\alpha} \mathrm{X}$-ray beam having the energy of $1253.3 \mathrm{eV}$ in the binding energy (B.E.) range of 10-1500 eV. The system was calibrated using the $84.0 \mathrm{eV}$ B.E. of the $\mathrm{Au} 4 \mathrm{f}_{7 / 2}$ line. The X-ray diffraction (XRD) D8 FOCUS, Bruker Ettlingen instrument with $\mathrm{Cu} \mathrm{K}_{\alpha}$ radiation $(\lambda=$ $1.5418 \AA$ ) operated at $30 \mathrm{~mA}$ and $40 \mathrm{kV}$ in the range of $5-80^{\circ}$ was used to study the diffraction pattern. The SHIMADZU, UV-VIS NIR 3600 spectrometer was used to obtain the absorption spectra of the samples in the wavelength region of 250-800 nm. To perform the desorption experiments, the N719 dye was desorbed from the unimplanted and Au-implanted $\mathrm{TiO}_{2}$ matrix using a $0.1 \mathrm{M}$ aqueous solution of potassium hydroxide $(\mathrm{KOH})$, and the absorbance spectra of the deloaded dye solutions were 

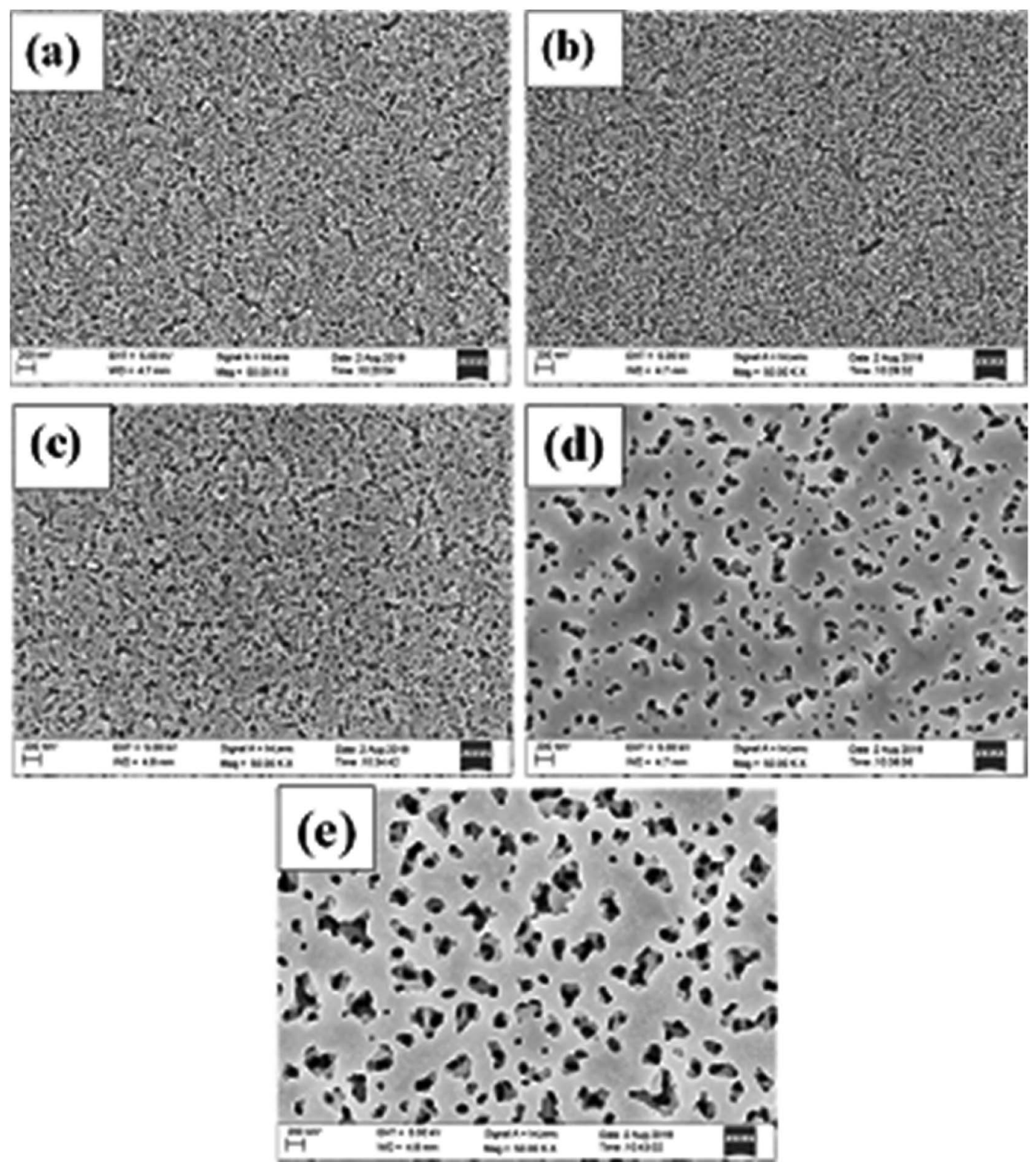

Fig. 1 FESEM images of the (a) unimplanted $\mathrm{TiO}_{2}$ and Au-implanted $\mathrm{TiO}_{2}$ at different fluence values: (b) F1, (c) F2, (d) F3 and (e) F4.

obtained. The photoluminescence (PL) spectra were studied using the PerkinElmer LS 55 Fluorescence Spectrometer within the 200-900 nm range at the excitation wavelength of $350 \mathrm{~nm}$. Raman spectroscopy was performed using a $514 \mathrm{~nm}$ argon laser source in the Renishaw InVia Reflex micro-Raman spectrometer in the wavenumber range of $30-1700 \mathrm{~cm}^{-1}$. Photovoltaic studies of the fabricated DSSCs were performed using a Keithley source meter (Model 2400) under 1 sun illumination at 1.5 G AM of intensity $100 \mathrm{~mW} \mathrm{~cm}^{-2}$ with an OAI, TriSOL solar simulator. The calibration of the solar simulator using a standard silicon cell was done prior to the measurements. The work function measurements of the plasmonic $\mathrm{TiO}_{2}$ were recorded using the Kelvin Probe technique (SKP, Kelvin Probe 4.5). Electrochemical impedance spectroscopy (EIS) measurements were performed using a frequency response analyzer (FRA) attached to an
Autolab potentiostat/galvanostat (PGSTAT12) in the frequency range from $0.01 \mathrm{~Hz}$ to $1 \mathrm{MHz}$.

\section{Results and discussion}

Fig. 1 shows the FESEM images of the unimplanted and Auimplanted $\mathrm{TiO}_{2}$ at different fluence values (F1, F2, F3 and F4). A porous surface is observed for $\mathrm{TiO}_{2}$, which seems to flatten with the implantation of Au. Furthermore, the flattening increased with an increase in fluence from F1 to F4. Consequently, the Au nps implanted in $\mathrm{TiO}_{2}$ up to a $22 \mathrm{~nm}$ depth were covered with a very thin layer of $\mathrm{TiO}_{2}$ melt according to the thermal spike model; ${ }^{42}$ the thin coverage of the $\mathrm{TiO}_{2}$ melt around the $\mathrm{Au}$ nps prevented their direct contact with the dye molecules and electrolyte. Moreover, the surface flattening led 


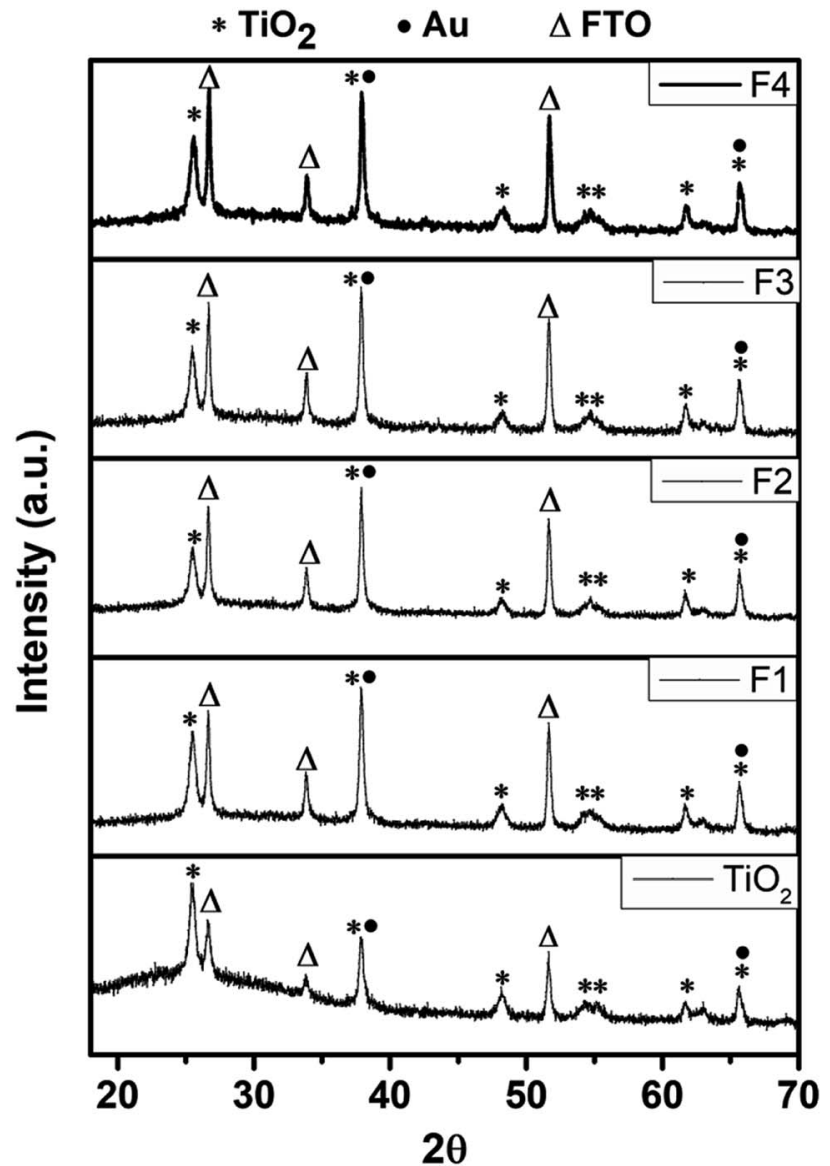

Fig. 2 XRD patterns of the unimplanted and $\mathrm{Au}$-implanted $\mathrm{TiO}_{2}$ matrix (F1, F2, F3 and F4).

to the widening of the pore size and decreased the number of pores.

The XPS survey spectra of the unimplanted and Auimplanted $\mathrm{TiO}_{2}$ (Fig. $\mathrm{S} 1 \dagger$ ) exhibit the presence of the $\mathrm{Ti}, \mathrm{O}$

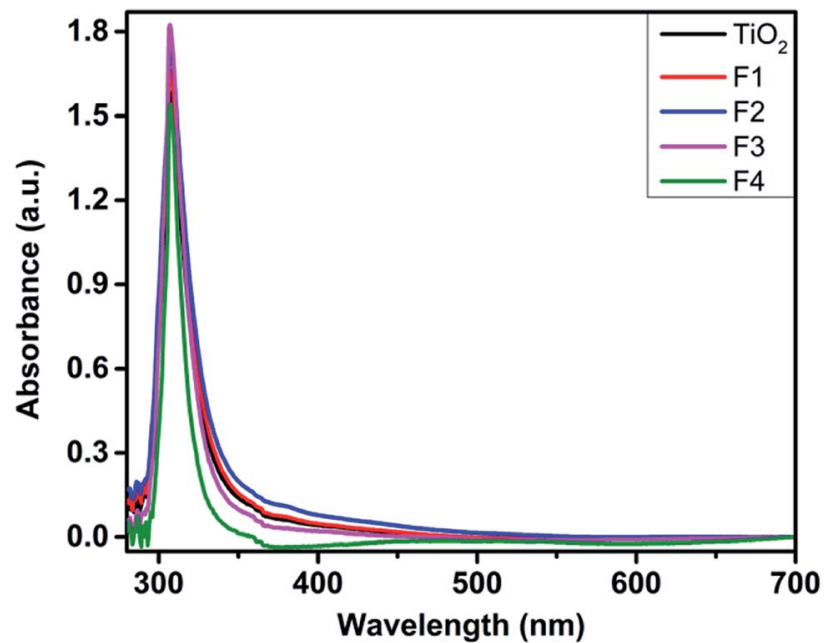

Fig. 3 UV-Vis absorption spectra of the N719 dye-loaded unimplanted and $\mathrm{Au}$-implanted $\mathrm{TiO}_{2}$ (F1, F2, F3 and F4) photoanodes.

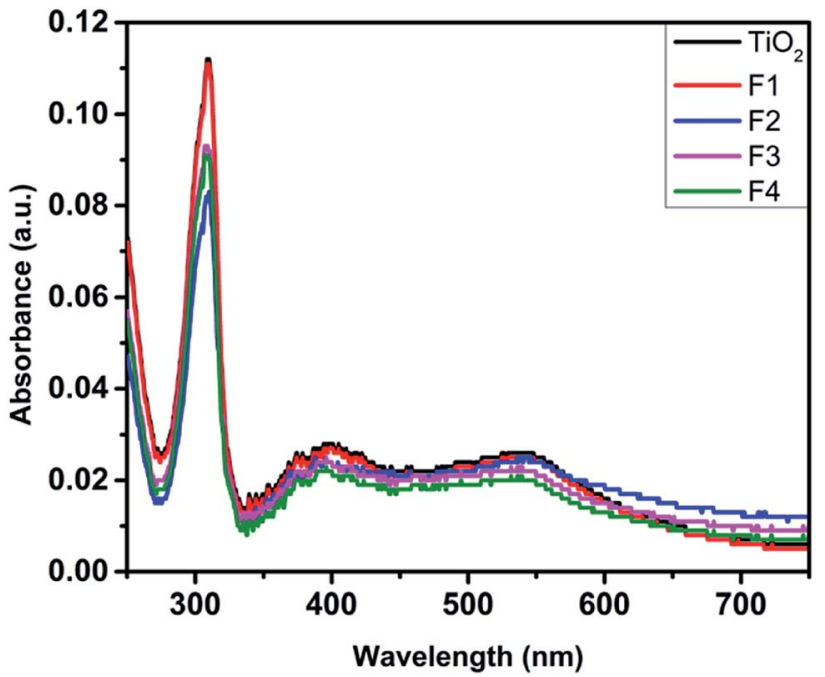

Fig. 4 UV-Vis absorption spectra of the N719 dye deloaded from the unimplanted and $\mathrm{Au}$-implanted $\mathrm{TiO}_{2}$ (F1, F2, F3 and F4) photoanodes.

Table 1 Dye desorption parameters of the unimplanted and Auimplanted photoanodes

\begin{tabular}{lll}
\hline Sample & $\begin{array}{l}\text { Concentration } \\
\text { of dye }(\mu \mathrm{M})\end{array}$ & $\begin{array}{l}\text { Dye loading }\left(\times 10^{-8} \mathrm{~mol}\right. \\
\left.\mathrm{cm}^{-2}\right)\end{array}$ \\
\hline $\mathrm{TiO}_{2}$ & 1.91 & 2.29 \\
$\mathrm{~F} 1$ & 1.83 & 2.19 \\
$\mathrm{~F} 2$ & 1.76 & 2.11 \\
$\mathrm{~F} 3$ & 1.68 & 2.02 \\
$\mathrm{~F} 4$ & 1.53 & 1.84
\end{tabular}

and $\mathrm{Au}$ elements. The elemental compositions of $\mathrm{Ti}, \mathrm{O}$ and $\mathrm{Au}$ present in all the samples were determined from the area under the curves in the core level XPS spectra of Ti-2p (Fig. S2 $\dagger$ ), O-1s (Fig. S3 $\dagger$ ) and Au-4f (Fig. S4†), respectively. The unimplanted $\mathrm{TiO}_{2}$ contained 0.28 and 0.72 atomic \%ages of $\mathrm{Ti}$ and $\mathrm{O}$, respectively, with the $\mathrm{Ti} / \mathrm{O}$ ratio of 0.6 . In contrast, the

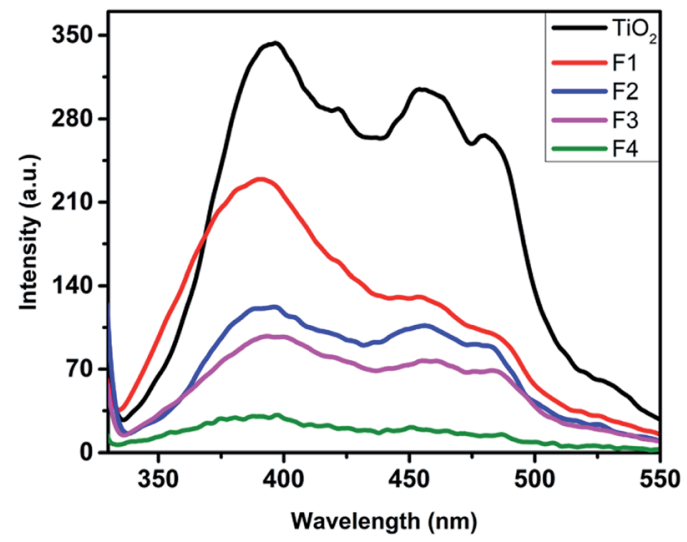

Fig. $5 \mathrm{PL}$ spectra of the unimplanted and Au-implanted $\mathrm{TiO}_{2}(\mathrm{~F} 1, \mathrm{~F} 2$, F3 and F4). 


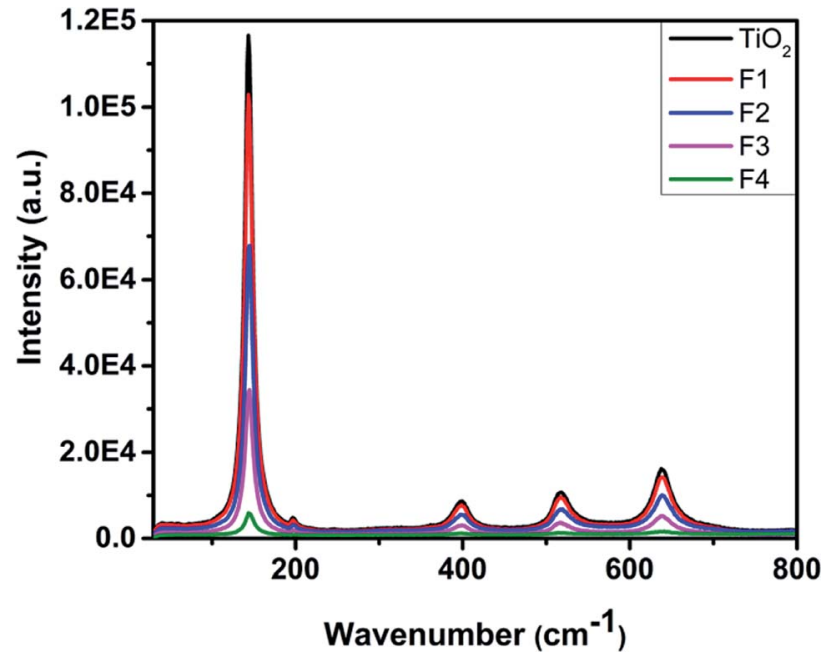

Fig. 6 Raman spectra of $\mathrm{TiO}_{2}$ and the Au-implanted $\mathrm{TiO}_{2}$ (F1, F2, F3 and F4).

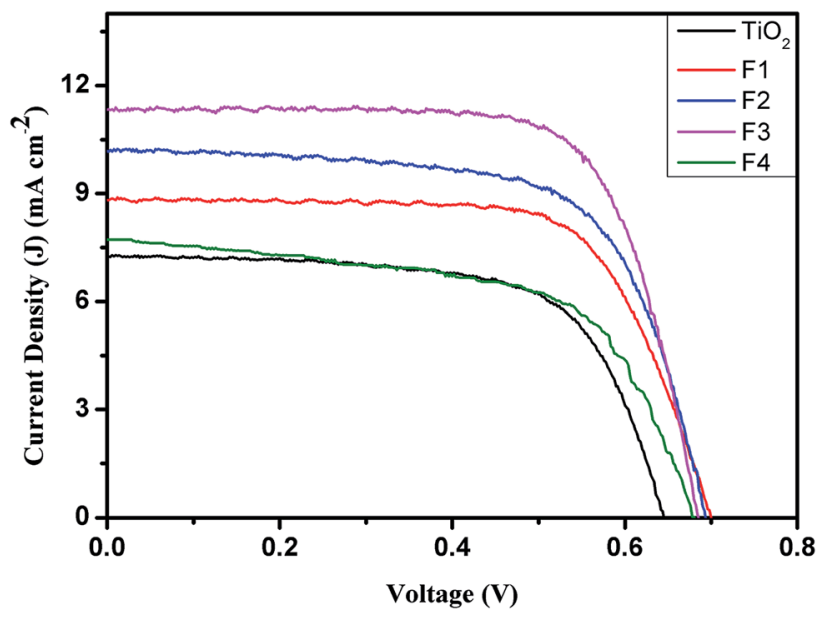

Fig. $7 \mathrm{~J}-V$ characteristics of the unimplanted and $\mathrm{Au}$-implanted $\mathrm{TiO}_{2}-$ based DSSCs.

implanted $\mathrm{TiO}_{2}$ showed the presence of the additional $\mathrm{Au}$ element, and its composition increased with an increase in $\mathrm{Au}$ fluence i.e. F1 (0.04\%), F2 (0.09\%), F3 (0.11\%) and F4 (0.42\%).

The XRD patterns of the $\mathrm{TiO}_{2}$ and Au-implanted $\mathrm{TiO}_{2}$ were compared, as shown in Fig. 2. The peaks at $25.49^{\circ}, 38.00^{\circ}$, $48.17^{\circ}, 54.58^{\circ}, 55.00^{\circ}, 61.58^{\circ}$ and $65.63^{\circ}$ are respectively assigned to the (101), (004), (200), (105), (211), (204) and (116) crystal planes of anatase $\mathrm{TiO}_{2}$ (JCPDS card no. 21-1272). ${ }^{23,43}$ In addition, the peaks at $26.64^{\circ}, 33.84^{\circ}$ and $51.57^{\circ}$ corresponding to the (201), (130) and (312) planes of the FTO substrates (JCPDS card no. 00-030-1375) ${ }^{44}$ were observed. The Au-implanted $\mathrm{TiO}_{2}$ exhibits similar peaks; however, the intensity of the peaks corresponding to the $2 \theta$ values of $38.00^{\circ}$ and $65.63^{\circ}$ increases with the increasing fluence due to the overlapping ${ }^{45}$ of the additional (111) and (220) planes of the face-centered cubic Au (JCPDS card no. 04-0784), ${ }^{43,46}$ respectively. Moreover, the intensity of the peaks at $26.64^{\circ}, 33.84^{\circ}$ and $51.57^{\circ}$ increases in the implanted $\mathrm{TiO}_{2}$ due to an increase in the pore size after Au implantation, thus providing more reflections of X-rays from the FTO surface. The sharp and well-defined diffraction peaks confirm the polycrystalline nature of the unimplanted and implanted $\mathrm{TiO}_{2}$.

Fig. 3 depicts the UV-Vis absorption spectra of the N719 dyeloaded unimplanted and Au-implanted $\mathrm{TiO}_{2}$ photoanodes. An absorption band around $307 \mathrm{~nm}$ is observed in the spectra of the unimplanted and Au-implanted $\mathrm{TiO}_{2}$ photoanodes, ascribed to the intrinsic inter-band exciton transitions. ${ }^{16,18}$ The characteristic absorption bands of the N719 dye around 390 and $530 \mathrm{~nm}$ were not observed for all the samples as these peaks became suppressed because of the high absorbance at $307 \mathrm{~nm}$. A gradual increase and broadening in the absorbance was observed up to the F3 photoanode, ascribed to the enhanced light absorption of sensitized dye molecules due to the coupling of a strong local field of LSPR around Au and the dipole moment of the dye molecules. Furthermore, the amount of the sensitized dye in the unimplanted and Au-implanted $\mathrm{TiO}_{2}$ was determined through dye desorption experiments by obtaining the absorbance spectra of the N719 dye deloaded from the samples (Fig. 4). The N719 dye molecules showed an absorbance around 390 and $530 \mathrm{~nm}$ in all the samples. The amount of dye loading was calculated as follows: ${ }^{47}$

Dye loading $\left(\mathrm{mol} \mathrm{cm}^{-2}\right)$

$$
=\frac{[\text { dye concentration }(\mathrm{M}) \times \text { volume }(\mathrm{ml})]}{\text { electrode area }\left(\mathrm{cm}^{2}\right)}
$$

where the dye concentration was measured using the Beer Lambert's law ${ }^{47}$ at the absorbance value of around $535 \mathrm{~nm}$. The concentration and amount of dye loading are tabulated in Table 1. At the lower fluence of up to $10^{15}$ ions $\mathrm{cm}^{-2}$ (F3), a small change in dye loading was observed as compared to the case of the comparatively higher fluence of $10^{16}$ ions $\mathrm{cm}^{-2}$ (F4). These observations are in good agreement with the FESEM studies, where the porosity was found to decrease appreciably at higher

Table 2 Photovoltaic, $\phi$ and EIS parameters of DSSCs fabricated with unimplanted and Au-implanted TiO ${ }_{2}$-based DSSCs

\begin{tabular}{lclllrr}
\hline Photoanodes & $J_{\text {SC }}\left(\mathrm{mA} \mathrm{cm}^{-2}\right)$ & $V_{\text {OC }}(\mathrm{V})$ & F.F. & PCE $(\%)$ & $R_{\mathrm{S}}\left(\Omega \mathrm{cm}^{2}\right)$ & $R_{2}\left(\Omega \mathrm{cm}^{2}\right)$ \\
\hline $\mathrm{TiO}_{2}$ & 7.23 & 0.65 & 0.65 & 3.09 & 8.47 & 8.57 \\
$\mathrm{~F} 1$ & 8.80 & 0.70 & 0.75 & 4.72 & 8.87 & 8.34 \\
$\mathrm{~F} 2$ & 10.15 & 0.69 & 0.68 & 4.84 & 9.60 & 9.87 \\
$\mathrm{~F} 3$ & 11.29 & 0.69 & 0.71 & 5.59 & 9.90 & 12.40 \\
$\mathrm{~F} 4$ & 7.71 & 0.69 & 0.59 & 3.15 & 10.10 & 5.25 \\
& & & & & 5.22 \\
\end{tabular}



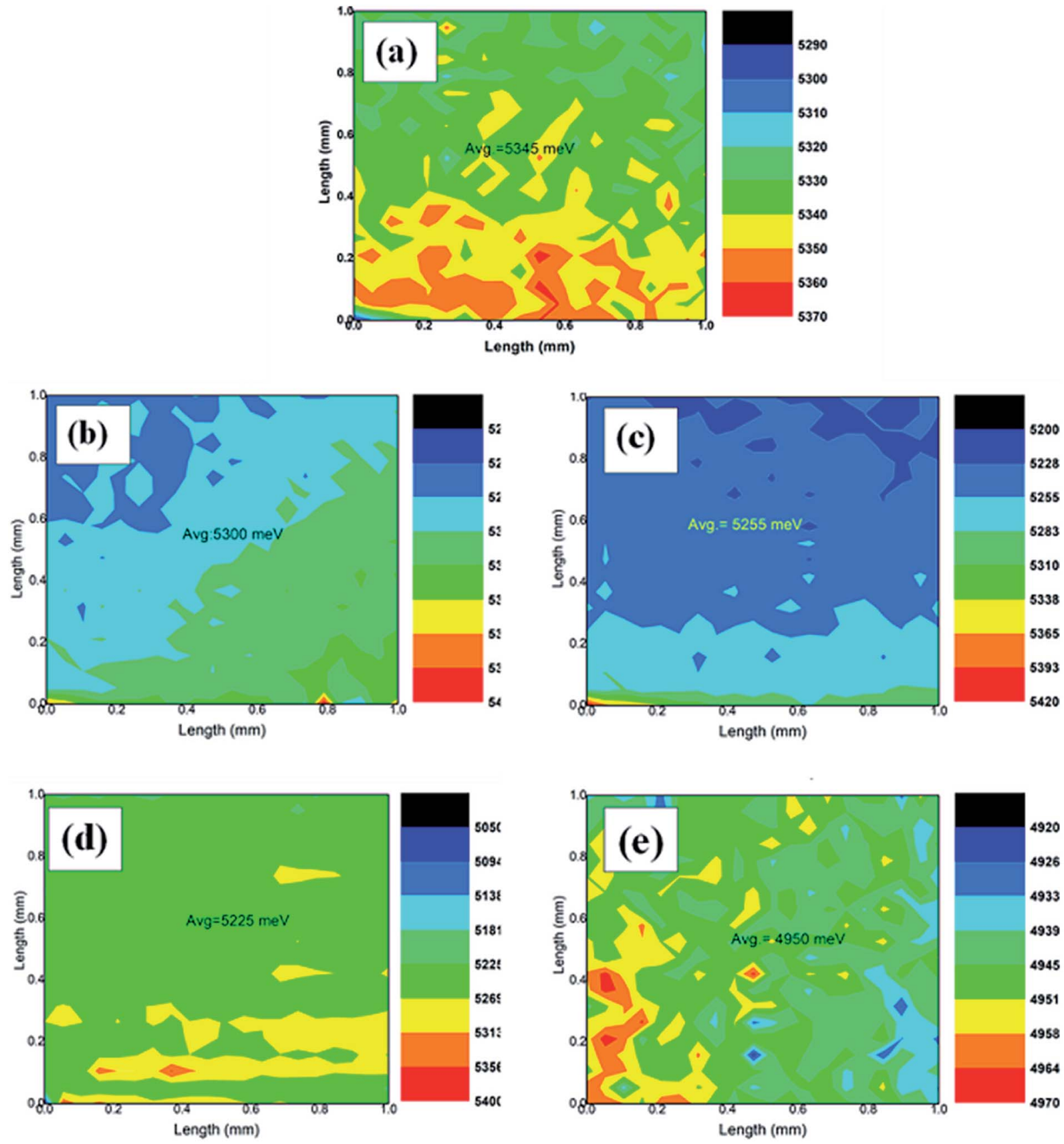

Fig. 8 Work function of (a) $\mathrm{TiO}_{2}$, (b) F1, (c) F2, (d) F3 and (e) F4.

fluence. Thus, the reduction in absorbance for the F4 photoanode (Fig. 3) is directly linked with a decrease in dye loading. Hence, the LSPR effect of Au enhances the absorbance but at the expense of dye loading; thus, their balanced effect should be optimized for efficient light harvesting.

The PL spectra of the unimplanted and Au-implanted photoanodes (Fig. 5) were obtained with $\lambda_{\text {excitation }}$ at $350 \mathrm{~nm}$ using a xenon lamp. The unimplanted $\mathrm{TiO}_{2}$ exhibits broad emission peaks at around 395 and $452 \mathrm{~nm}$ corresponding to the transitions involving the recombination of electrons trapped at levels within the forbidden band gap of $\mathrm{TiO}_{2}$ arising due to the intrinsic oxygen vacancies. ${ }^{21,48}$ The Au-implanted $\mathrm{TiO}_{2}$ exhibits similar emission spectra, and its PL intensities are lower than those of the unimplanted $\mathrm{TiO}_{2}$. Furthermore, it is observed that the PL intensity decreases with an increase in fluence (from F1 to F4). The photoluminescence occurs due to the emission of photons by the recombination of photo-generated electrons and holes, which infers a lower recombination rate for the lowerintensity peaks. Thus, the plasmonic $\mathrm{TiO}_{2}$ exhibits a lower charge recombination rate, which is least for the F4 sample. It can be explained on the basis of the formation of Schottky barriers ${ }^{48,49}$ at the $\mathrm{TiO}_{2}$ and $\mathrm{Au}\left(\mathrm{TiO}_{2} @ \mathrm{Au}\right)$ interface that help to capture the photo-generated electrons before they recombine with holes by accumulating them around Au. Thus, Au acts as 
(a)
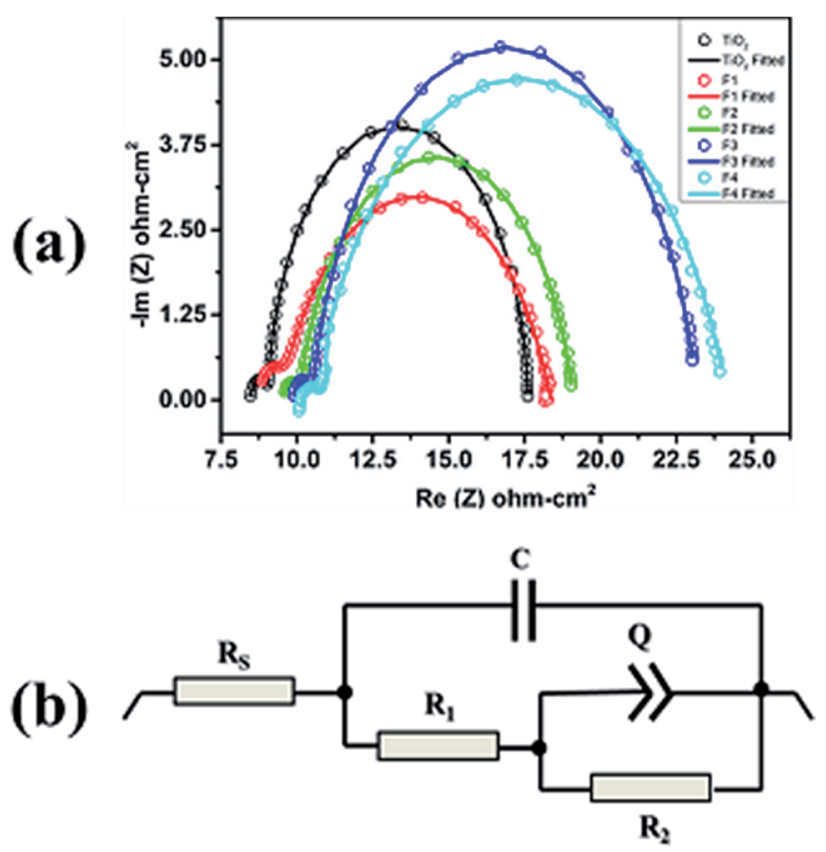

Fig. 9 (a) EIS of DSSCs fabricated with the unimplanted and Auimplanted $\mathrm{TiO}_{2}$-based DSSCs (F1, F2, F3 and F4); and (b) their equivalent circuit model where $R_{\mathrm{S}}, R_{1}$, and $R_{2}$ have their respective meanings, $Q$ is the constant phase element and $C$ is the capacitance.

a charge separation center for the photo-generated electrons. ${ }^{45}$ Moreover, the lifetime of the photo-generated electrons increases (discussed later in the EIS studies); this improves the electron charge transport and inhibits the electron-hole recombination process.

Fig. 6 shows the Raman spectra of the unimplanted and Auimplanted $\mathrm{TiO}_{2}$ obtained at room temperature using a $514 \mathrm{~nm}$ argon laser source. The peaks of the unimplanted anatase phase of $\mathrm{TiO}_{2}$ are observed at 145, 200, 403, 520 and $643 \mathrm{~cm}^{-1}$, corresponding to the $\mathrm{E}_{\mathrm{g}(1)}, \mathrm{E}_{\mathrm{g}(2)}, \mathrm{B}_{1 \mathrm{~g}(1)}, \mathrm{A}_{1 \mathrm{~g}(1)}+\mathrm{B}_{1 \mathrm{~g}(2)}$ and $\mathrm{E}_{\mathrm{g}(3)}$ Raman-active phonon modes. The highly intense Raman peak at $145 \mathrm{~cm}^{-1}$ is closely related to the anatase $\mathrm{TiO}_{2}$ single crystal. ${ }^{\mathbf{5 0}}$ The Au-implanted $\mathrm{TiO}_{2}$ exhibits similar Raman peaks; however, a gradual decrease in their intensities is observed with an increase in fluence; this infers an increase in the number of defects on the $\mathrm{TiO}_{2}$ surface created by the Schottky barriers. ${ }^{45} \mathrm{Au}$ does not possess any specific phonon mode because of its weak Raman scattering power; this suggests that the Au implantation does not alter the phase of $\mathrm{TiO}_{2}$.

The current density-voltage $(J-V)$ characteristics of DSSCs fabricated with the unimplanted and Au-implanted $\mathrm{TiO}_{2}$ are shown in Fig. 7. The obtained photovoltaic parameters, such as short circuit current density $\left(J_{\mathrm{SC}}\right)$, open circuit voltage $\left(V_{\mathrm{OC}}\right)$, fill factor (F.F.) and PCE, summarized in Table 2, signify the beneficial effect of $\mathrm{Au}$ implantation on the photovoltaic performance of the DSSCs. The $J_{\mathrm{SC}}$ of the Au-implanted $\mathrm{TiO}_{2}$ based DSSC is found to be greater than that of the unimplanted $\mathrm{TiO}_{2}$-based DSSC due to the enhanced light absorption owing to the LSPR of Au. Furthermore, the $J_{\mathrm{SC}}$ increases with the increasing fluence up to the F3-based DSSC, and then, a sudden decrease in the $J_{\mathrm{SC}}$ is observed due to the decreased dye loading onto the $\mathrm{TiO}_{2}$ surface. Although similar values of $V_{\mathrm{OC}}$ are

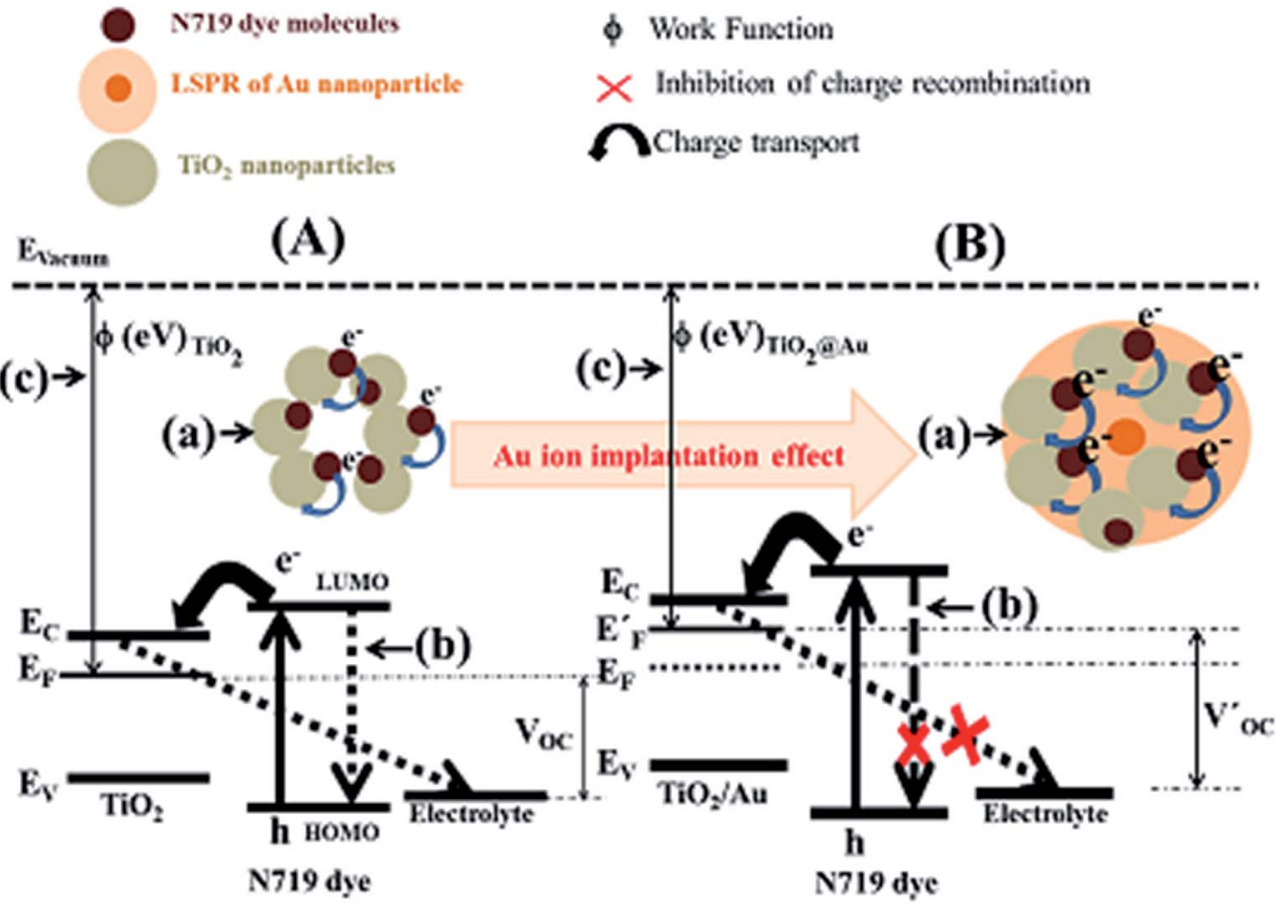

Fig. 10 Schematic of the working mechanism of the unimplanted (A) and Au-implanted (B) plasmonic DSSCs highlighting the enhanced light harvesting $(A(a)$ and $B(a))$, back recombination inhibition of charge carriers $(A(b)$ and $B(b))$ and $E_{F}$-shifting $(A(c)$ and $B(c))$ upon the implantation of Au. 
obtained for the plasmonic DSSCs, they are comparatively higher than those of the unimplanted DSSC; this has been explained by the work function measurements. The surface work function $\phi$ of the unimplanted $\mathrm{TiO}_{2}$ (Fig. 8(a)) has been found to be $5.34 \mathrm{eV}$, which further decreases upon $\mathrm{Au}$ implantation (Fig. 8(b-e), Table 2). This indicates an upward shift in $E_{\mathrm{F}}$ induced by the Schottky barriers. ${ }^{51}$ The upward shift in the $E_{\mathrm{F}}$ values of the plasmonic $\mathrm{TiO}_{2}$ confirms the increase in $V_{\mathrm{OC}}$ as it is defined as the difference between the $E_{\mathrm{F}}$ of the photoanode and the redox potential of the electrolyte (constant herein). The comparatively larger change in the $J_{\mathrm{SC}}$ than that in $V_{\mathrm{OC}}$ contributes to the enhancement in the PCEs of the implanted DSSCs (Table 2). Thus, the PCEs of the plasmonic DSSCs are found to be higher than those of the unimplanted DSSCs. Furthermore, the PCE increases with an increase in fluence up to $10^{15}$ ions $\mathrm{cm}^{-2}$ and suddenly decreases for the F4-based DSSC. Hence, the F3-based DSSC exhibits the maximum PCE of $5.59 \%$ with an increment of $44.7 \%$ as compared to the $\mathrm{TiO}_{2}$ based DSSC (3.09\%).

The EIS measurements were performed on the unimplanted and $\mathrm{Au}$-implanted DSSCs to understand the variation in $J_{\mathrm{SC}}$ and reduced electron-hole recombination rate through the interfacial electronic charge transfer process. The Nyquist plots (Fig. 9(a)) were obtained for all the DSSCs operated at their respective $V_{\mathrm{OC}}$ under illumination and showed two well-defined semicircles. The Nyquist plots fitted with the equivalent circuit model (Fig. 9(b)) provide an estimated value of the total DC resistance comprising the combination of $R_{\mathrm{S}}, R_{1}$ and $R_{2}$. Herein, $R_{1}$ corresponds to the electron charge transfer resistance at the $\mathrm{CE} /$ electrolyte interface and is determined from the diameter of the first semicircle in the Nyquist plot; moreover, it is almost same for all the DSSCs as the CE is similar in all the devices. The intercept of the high frequency semicircle at the real axis provides the $R_{\mathrm{S}}$ value representing the ohmic series resistance influenced by FTO, $\mathrm{TiO}_{2}$ (with and without Au nps), Pt CE, and the electrolyte. ${ }^{19,45} R_{\mathrm{S}}$ increases slightly for the Au-implanted DSSCs as compared to that for the unimplanted DSSC (Table 2) due to an additional resistance offered by the Schottky barriers at the $\mathrm{TiO}_{2} @ \mathrm{Au}$ interface. Furthermore, the $R_{\mathrm{S}}$ values show a continuous increase with the increasing fluence (from F1 to F4) due to the presence of more Schottky barriers as the Au content increases in $\mathrm{TiO}_{2}$. The diameter of the Nyquist second semicircle depicts the charge transfer resistance $\left(R_{2}\right)$ at the unimplanted and Au-implanted $\mathrm{TiO}_{2} @ \mathrm{~N} 719 /$ electrolyte interface. ${ }^{19}$ The values of $R_{2}$ for the Au-implanted DSSCs have been found to be higher than those for the unimplanted DSSCs because of the charge separation ability of $\mathrm{Au}$ in the Schottky barriers, ${ }^{21}$ which prevents the electrons from recombining with the holes at the highest occupied molecular orbital (HOMO) of the N719 dye. Moreover, $R_{2}$ increases with the increasing fluence; this leads to a reduced electron recombination rate that further contributes to the increased $J_{\mathrm{SC}}$ values (Table 2). Although $R_{2}$ is maximum and the electron hole recombination rate for F4 is least, $J_{\mathrm{SC}}$ still decreases for the F4 DSSC because the lower dye-loading generates a lower number of charge carriers for transport throughout the circuit. Thus, the F3-based DSSC with the increased values of $R_{\mathrm{S}}$ and $R_{2}$ exhibits a significantly reduced recombination, resulting in the highest fill factor of 0.71 (Table 2).

Moreover, the electron-hole recombination process at the $\mathrm{TiO}_{2} @$ N719/electrolyte interface has been supported through the electron lifetime $(\tau)$ measurements. It is observed that the maxima of the characteristic maximum frequency $\left(f_{\max }\right)$ of the Nyquist $2^{\text {nd }}$ semicircle shifts towards a lower frequency region with an increase in the Au fluence from the F1 to F4 DSSCs as compared to the case of the unimplanted DSSC. This reveals longer electron lifetime as it is inversely proportional to $f_{\max }$, i.e., $\tau=\frac{1}{2 \pi f_{\max }}$, which reduces the electron-hole recombination rate due to the increased $\mathrm{Au}$ content in $\mathrm{TiO}_{2}$, characterized by larger $R_{2}$ values.

On the basis of the abovementioned morphological, compositional, spectroscopic, $J-V$ and EIS investigations, a plausible mechanism behind the improvement of the PCE of Au-implanted plasmonic DSSCs as compared to that of the unimplanted DSSC has been explained (Fig. 10).

Compared to the Au-implanted photoanodes, a comparatively lower number of photo-generated electrons get excited from the HOMO to LUMO level of the N719 dye (Fig. 10A(a) and $\mathrm{B}(\mathrm{a}))$. Some of these electrons recombine with the dye molecules as well as the electrolyte (Fig. 10A(b)) instead of entering the conduction band of $\mathrm{TiO}_{2}$. The lower photo-generated electrons and higher recombination rate resulted in smaller $J_{\mathrm{SC}}$ and $V_{\mathrm{OC}}$ (Fig. 10A(c)) and consequently smaller FF and PCE of the unimplanted DSSC. In contrast, the plasmonic DSSCs with implanted photoanodes exhibit a comparatively higher number of photo-generated electrons upon absorption of more photons by the N719 dye molecules due to the LSPR effect (Fig. 10B(a)). Further, the formation of the Schottky barriers at the $\mathrm{TiO}_{2} @ \mathrm{Au}$ interface inhibits the back recombination of electrons (Fig. $10 \mathrm{~B}(\mathrm{~b})$ ) to provide a comparatively higher $J_{\mathrm{SC}}$. Moreover, the decrease in $\phi$ of $\mathrm{TiO}_{2}$ upon implantation (Fig. 10B(c)) shifts the $E_{\mathrm{F}}$ towards an upward direction and results in an increase of the $V_{\mathrm{OC}}$. Hence, the increase of both $J_{\mathrm{SC}}$ and $V_{\mathrm{OC}}$ upon implantation results in the improved FF and PCE of the plasmonic DSSCs.

In addition, the photovoltaic parameters improve with the increasing fluence of $\mathrm{Au}$ implantation (Table 2) due to comparatively more photo-generated electrons, back recombination inhibition and a decrease in $\phi$ of $\mathrm{TiO}_{2}$. However, the PCE of the F4-based DSSC is found to be limited by the amount of dye-loading content as the porosity of $\mathrm{TiO}_{2}$ decreases at higher fluence, which further reduces the dye-loading.

\section{Conclusion}

The electronic and optical properties of $\mathrm{TiO}_{2}$ photoanodes were successfully tuned through Au implantation for the fabrication of highly stable and efficient plasmonic DSSCs. The DSSCs containing $\mathrm{TiO}_{2}$ implanted with 0.11 atomic \%age of Au under the fluence of $10^{15}$ ions $\mathrm{cm}^{-2}$ showed long-term stability along with improved photovoltaic performance, having the PCE, $J_{\mathrm{SC}}$ and $V_{\mathrm{OC}}$ values of $5.59 \%, 11.29 \mathrm{~mA} \mathrm{~cm}^{-2}$ and $0.69 \mathrm{~V}$ as compared to the $\mathrm{TiO}_{2}$-based DSSCs $\left(\mathrm{PCE}=3.09 \%, J_{\mathrm{SC}}=7.23 \mathrm{~mA}\right.$ 
$\mathrm{cm}^{-2}$ and $V_{\mathrm{OC}}=0.65 \mathrm{~V}$, respectively). The increment of $44.7 \%$ in the PCE of the plasmonic DSSCs is attributed to the enhanced light absorption and reduced electron hole recombination rate. Thus, ion implantation has the potential to fabricate stable as well as efficient plasmonic DSSCs than the DSSCs containing bare as well as core shell Mnps.

\section{Conflicts of interest}

There are no conflicts of interest to declare.

\section{Acknowledgements}

The authors are thankful to Inter University Accelerator Centre, New Delhi, for providing financial support through Project No. UFR-60331. One of the authors, Navdeep Kaur, is thankful to UGC, New Delhi, India, for providing financial assistance in support of the present research work.

\section{References}

1 Q. Hao, C. Wang, H. Huang, W. Li, D. Du, D. Han, T. Qiu and P. K. Chu, Sci. Rep., 2015, 5, 15288.

2 Y. Wang, L. Liu, L. Xu, C. Meng and W. Zhu, J. Appl. Phys., 2013, 113, 174311.

3 S. T. Nishanthi, S. Iyyapushpam, B. Sundarakannan, E. Subramanian and D. Pathinettam Padiyan, J. Power Sources, 2015, 274, 885-893.

4 S. Hirose, S. Ueda and N. Ohashi, J. Appl. Phys., 2019, 125, 095301.

5 P. Zimmermann, A. Hötger, N. Fernandez, A. Nolinder, K. Müller, J. J. Finley and A. W. Holleitner, Nano Lett., 2019, 19, 1172-1178.

6 Y. Shao, X. Li, L. Wu and D. Wang, J. Appl. Phys., 2018, 125, 013101.

7 J. Luo, J. Chen, H. Wang and H. Liu, J. Power Sources, 2016, 303, 287-293.

8 H. Wang, F. Chen, W. Li and T. Tian, J. Power Sources, 2015, 287, 150-157.

9 N. Okada, N. Morishita, A. Mori, T. Tsukada, K. Tateishi, K. Okamoto and K. Tadatomo, J. Appl. Phys., 2017, 121, 153102.

10 T. Plint, B. H. Lessard and T. P. Bender, J. Appl. Phys., 2016, 119, 145502.

11 O. A. Yeshchenko, S. Z. Malynych, S. O. Polomarev, Y. Galabura, G. Chumanov and I. Luzinov, RSC Adv., 2019, 9, 8498-8506.

12 P. Halkare, N. Punjabi, J. Wangchuk, A. Nair, K. Kondabagil and S. Mukherji, Sens. Actuators, B, 2019, 281, 643-651.

13 N. J. Hogan, A. S. Urban, C. Ayala-Orozco, A. Pimpinelli, P. Nordlander and N. J. Halas, Nano Lett., 2014, 14, 46404645.

14 J. Kim, Lab Chip, 2012, 12, 3611-3623.

15 Tanvi, A. Mahajan, R. K. Bedi, S. Kumar, V. Saxena, A. Singh and D. K. Aswal, RSC Adv., 2016, 6, 48064-48071.

16 W. Hou, P. Pavaskar, Z. Liu, J. Theiss, M. Aykol and S. B. Cronin, Energy Environ. Sci., 2011, 4, 4650-4655.
17 B. O'Regan and M. Grätzel, Nature, 1991, 353, 737.

18 Y. H. Jang, Y. J. Jang, S. T. Kochuveedu, M. Byun, Z. Lin and D. H. Kim, Nanoscale, 2014, 6, 1823-1832.

19 S. Chang, Q. Li, X. Xiao, K. Y. Wong and T. Chen, Energy Environ. Sci., 2012, 5, 9444-9448.

20 Y. Wang, J. Zhai and Y. Song, RSC Adv., 2015, 5, 210-214.

21 H. Dong, Z. Wu, A. El-Shafei, B. Xia, J. Xi, S. Ning, B. Jiao and

X. Hou, J. Mater. Chem. A, 2015, 3, 4659-4668.

22 Y. Li, H. Wang, Q. Feng, G. Zhou and Z.-S. Wang, Gold nanoparticles inlaid $\mathrm{TiO}_{2}$ photoanodes: a superior candidate for high-efficiency dye-sensitized solar cells, 2013.

23 H.-W. Chen, C.-Y. Hong, C.-W. Kung, C.-Y. Mou, K. C. W. Wu and K.-C. Ho, J. Power Sources, 2015, 288, 221-228.

24 S. Muduli, O. Game, V. Dhas, K. Vijayamohanan, K. A. Bogle, N. Valanoor and S. B. Ogale, Sol. Energy, 2012, 86, 1428-1434. 25 N. Chander, A. F. Khan, E. Thouti, S. K. Sardana, P. S. Chandrasekhar, V. Dutta and V. K. Komarala, Sol. Energy, 2014, 109, 11-23.

26 L. Zhao, C. Zhong, Y. Wang, S. Wang, B. Dong and L. Wan, J. Power Sources, 2015, 292, 49-57.

27 H. Ran, J. Fan, X. Zhang, J. Mao and G. Shao, Enhanced performances of dye-sensitized solar cells based on $\mathrm{Au}-\mathrm{TiO}_{2}$ and $\mathrm{Ag}-\mathrm{TiO}_{2}$ plasmonic hybrid nanocomposites, 2017.

28 Y. Li, H. Wang, Q. Feng, G. Zhou and Z.-S. Wang, Energy Environ. Sci., 2013, 6, 2156-2165.

29 C. Hägglund, M. Zäch and B. Kasemo, Appl. Phys. Lett., 2008, 92, 013113.

30 Tanvi, A. Mahajan, R. K. Bedi, S. Kumar, V. Saxena and D. K. Aswal, J. Appl. Phys., 2015, 117, 083111.

31 S. D. Standridge, G. C. Schatz and J. T. Hupp, J. Am. Chem. Soc., 2009, 131, 8407-8409.

32 H. Choi, W. T. Chen and P. V. Kamat, ACS Nano, 2012, 6, 4418-4427.

33 Y.-Z. Zheng, X. Tao, J.-W. Zhang, X.-S. Lai and N. Li, J. Power Sources, 2018, 376, 26-32.

34 L. Bai, M. Li, K. Guo, M. Luoshan, H. F. Mehnane, L. Pei, M. Pan, L. Liao and X. Zhao, J. Power Sources, 2014, 272, 1100-1105.

35 S. D. Standridge, G. C. Schatz and J. T. Hupp, Langmuir, 2009, 25, 2596-2600.

36 J. Du, J. Qi, D. Wang and Z. Tang, Energy Environ. Sci., 2012, 5, 6914-6918.

37 Q. Xu, F. Liu, Y. Liu, K. Cui, X. Feng, W. Zhang and Y. Huang, Sci. Rep., 2013, 3, 2112.

38 F. Singh, S. Mohapatra, J. P. Stoquert, D. K. Avasthi and J. C. Pivin, Presented in part at the The Seventh International Symposium on Swift Heavy Ions in Matter, Lyon, France, 2008.

39 Y. K. Mishra, F. Singh, D. K. Avasthi, J. C. Pivin, D. Malinovska and E. Pippel, Appl. Phys. Lett., 2007, 91, 063103.

40 A. Kumar, M. K. Jaiswal, D. Kanjilal, R. K. Joshi and T. Mohanty, Appl. Phys. Lett., 2011, 99, 013109.

41 J. Luo, J. Zhou, H. Guo, W. Yang, B. Liao, W. Shi and Y. Chen, RSC Adv., 2014, 4, 56318-56322.

42 C. Dufour, V. Khomrenkov, Y. Y. Wang, Z. G. Wang, F. Aumayr and M. Toulemonde, J. Phys.: Condens. Matter, 2017, 29, 095001. 
43 L. Zhang and Z.-S. Wang, J. Mater. Chem. C, 2016, 4, 36143620.

44 M. A. Al-Azawi, N. Bidin, M. Bououdina and S. M. Mohammad, Sol. Energy, 2016, 126, 93-104.

45 S. P. Lim, A. Pandikumar, N. M. Huang and H. N. Lim, RSC Adv., 2015, 5, 44398-44407.

46 S. W. Sheehan, H. Noh, G. W. Brudvig, H. Cao and C. A. Schmuttenmaer, J. Phys. Chem. C, 2013, 117, 927-934. 47 V. Saxena, P. Veerender, A. K. Chauhan, P. Jha, D. K. Aswal and S. K. Gupta, Appl. Phys. Lett., 2012, 100, 133303.
48 M. C. Mathpal, P. Kumar, A. K. Tripathi, R. Balasubramaniyan, M. K. Singh, J. S. Chung and A. Agarwal, New J. Chem., 2015, 39, 6522-6530.

49 P. Nbelayim, G. Kawamura, W. Kian Tan, H. Muto and A. Matsuda, Sci. Rep., 2017, 7, 15690.

50 Y. L. Du, Y. Deng and M. S. Zhang, J. Phys. Chem. Solids, 2006, 67, 2405-2408.

51 A. Kumar, A. S. Patel and T. Mohanty, J. Phys. Chem. C, 2012, 116, 20404-20408. 